

\title{
Separation of Traveling Waves in Cortical Networks Using Optical Imaging
}

\author{
Nicolas Schmidt, Gabriel Peyré, Yves Frégnac, Per Roland
}

\section{To cite this version:}

Nicolas Schmidt, Gabriel Peyré, Yves Frégnac, Per Roland. Separation of Traveling Waves in Cortical Networks Using Optical Imaging. IEEE International Symposium on Biomedical Imaging (ISBI) 2010, Apr 2010, Rotterdam, Netherlands. pp.868-871, 10.1109/ISBI.2010.5490124 . hal-00450733

\section{HAL Id: hal-00450733 https://hal.science/hal-00450733}

Submitted on 26 Jan 2010

HAL is a multi-disciplinary open access archive for the deposit and dissemination of scientific research documents, whether they are published or not. The documents may come from teaching and research institutions in France or abroad, or from public or private research centers.
L'archive ouverte pluridisciplinaire HAL, est destinée au dépôt et à la diffusion de documents scientifiques de niveau recherche, publiés ou non, émanant des établissements d'enseignement et de recherche français ou étrangers, des laboratoires publics ou privés. 


\section{SEPARATION OF TRAVELING WAVES IN CORTICAL NETWORKS USING OPTICAL IMAGING}

\author{
Nicolas Schmidt, Gabriel Peyré * \\ Ceremade, UMR CNRS 7534 \\ Université Paris-Dauphine \\ Paris, France
}

\begin{abstract}
This paper introduces a mathematical model of the spatiotemporal patterns of visually evoked activity observed using Voltage-Sensitive Dye Imaging (VSDI) of the visual cortex. The cortical activity is described using a linear superposition of waves traveling with different speeds. This model improves the quality of the wave detection and still respects the previous approaches, as it integrates several biologically plausible constraints: 1) separability of the sources in terms of cortical location; 2) separability of the waves in terms of propagation speed, and 3) additivity of the depolarizing effects of the waves. Under these assumptions, a traveling component analysis algorithm performs a full separation of the set of waves and recovers the locations of the neural sources. Both features could help to better understand the dynamics of evoked activity in cortical sensory networks.
\end{abstract}

Index Terms - Optical imaging, Inverse problems, Propagation, Partial differential equations, Signal reconstruction.

\section{INTRODUCTION}

There is a one-to-one mapping of the world projection on the retina onto the cortical laminar sheet of the primary visual cortex. The cortical contribution to the emergence of non-attentive visual perception also involves the global processing of the whole scene by primary and secondary visual cortical areas, mediated by long-range horizontal connections and top-down influences from higher-cortical areas. The complexity of the evoked cortical patterns requires advanced processing technique to decode this activity.

\subsection{Voltage Sensitive Dye Imaging (VSDI)}

In VSDI, the dye molecules bind to the external surface of cell membranes and act as molecular transducers which transform changes in membranes potential into fluorescent optical signals [1]. These optical changes are monitored with lightimaging devices. This imaging technique offers the high-

\footnotetext{
* This work is supported by ANR grant NatImages (ANR-08-EMER-009)

$\dagger$ and FACETS (FET Bio-I3: 015879).
}

est spatial resolution (about $50 \mu \mathrm{m}$ ) and temporal resolution (about $1 \mu s$ ) for imaging at the mesoscopic level.

\subsection{Traveling Cortical Waves}

Forward wave. Several authors [2, 3, 4] have reported that the activity evoked in the primary visual cortex is characterized by a feedforward and lateral wave of depolarization originating from the retinotopic center of the stimulation. This wave is initiated in mammals by the retino-thalamocortical retinotopic projection and relayed intra V1 through horizontal connection extending over several $\mathrm{mm}$ in the plane of the superficial cortical layers.

Per Roland et al. [5] stained the visual cortex of the ferret with VSD and presented a short-duration high contrast square during $133 \mathrm{~ms}$, starting at time $200 \mathrm{~ms}$. Figure 1, top row, shows the time evolution of a signal $X(t, x)$ obtained by averaging optical signals through 10 trials. This shows the forward traveling wave that emanates from two source points that correspond to the same retinotopic center.

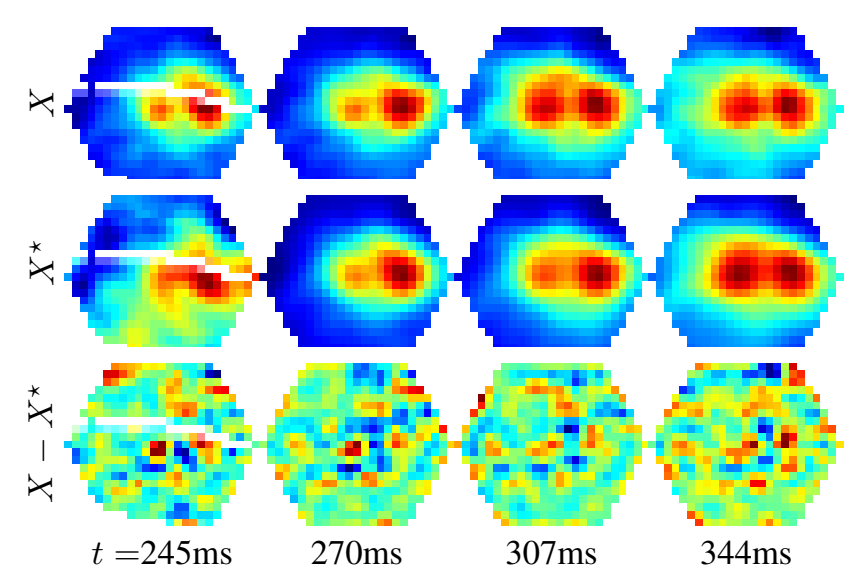

Fig. 1. Top row: optical signal $X$, for several times $t$ in ms. Middle row: reconstructed signal $X^{\star}$. Bottom row: residue $X^{\star}-X$. Values range from -0.0019 (blue) to 0.0155 (red)

Feedback wave. Per Roland et al show in [5] the existence of an other propagating wave of intracortical origin. This wave is interpreted as a feedback wave from higher vi- 
sual cortical areas going back to the primary visual cortex. It is shown in [5] that this feedback wave has several key characteristics that differ from the forward wave. In particular, it originates from a distinct secondary visual cortical area (corresponding a distinct representation of the visual field), travels faster and have a very small amplitude in comparison with the forward wave. This wave is extracted in [5] using a pointwise normalization of the input signal

$$
\tilde{\theta}_{2}(t, x)=\max \left(X(t, x)-\max _{t^{\prime} \in[0, T]} X\left(t^{\prime}, x\right), \tau\right)
$$

for a well chosen threshold $\tau<0$, and $\tilde{\theta}_{1}=X-\tilde{\theta}_{2}$ is the first wave, as shown in Figure 2, bottom row.

\subsection{Contributions}

This paper proposes a mathematical model obeying plausible biological constraints, for both forward and feedback waves observed in VSDI signal. This model defines the notion of traveling wave component, that enables to separate the waves and locate their sources using a numerical algorithm. This approach is more accurate than the normalization (1) because it integrates global propagation constraints, regularizes the signal and extracts the sources.

\section{MATHEMATICAL MODEL OF SIGNAL FORMATION}

\subsection{Optical Signal Formation Model}

Our model assumes that the VSDI signal is a linear superposition of several components

$$
X=\theta_{1}+\theta_{2}+W
$$

where $\theta_{1}$ corresponds to the forward wave, $\theta_{2}$ to the backward wave, and $W$ takes into account modeling imperfections and acquisition noise. The linear mixing (2) is a simplifying assumption that is coherent with optical recording and seems to fit reasonably with experimental observations [5, 1].

\subsection{Traveling Component Model}

Each propagating component $\theta_{i}$, for $i=1,2$, is assumed to be the solution of the non-homogenous wave equation

$$
\begin{array}{ll}
\frac{\partial^{2} \theta_{i}}{\partial t^{2}}-c_{i}^{2} \Delta \theta_{i}=f_{i} & \text { in } E=] 0, T\left[\times \mathbb{R}^{2}\right. \\
\theta_{i}=0, \frac{\partial \theta_{i}}{\partial t}=0 & \text { in }\{t=0\} \times \mathbb{R}^{2}
\end{array}
$$

where $f_{i}$ is the source of the component and $c_{i} \in \mathbb{R}^{+}$is a fixed speed.

This modeling hypothesis is a simple way to express the fact that cortical waves are traveling with an approximately constant speed but that the two speeds $c_{1}, c_{2}$ may be different.

For smooth enough sources $f_{i} \in \mathcal{C}^{2}(E)$, where $E=$ ] $0, T\left[\times \mathbb{R}^{2}\right.$, the equation (3) is known to have a unique solution. We denote as $\theta_{i}=T_{c_{i}}\left(f_{i}\right)$ the linear mapping from the sources to the propagating component. The optical data formation process (2) is rewritten as

$$
X=T_{c_{1}}\left(f_{1}\right)+T_{c_{2}}\left(f_{2}\right)+W .
$$

\section{SEPARATION OF MULTIPLE TRAVELING WAVES}

The separation problem requires to estimate two sources $\left(f_{1}^{\star}, f_{2}^{\star}\right)$ from the noisy observations $X$ alone, so that

$$
X \approx T_{c_{1}}\left(f_{1}^{\star}\right)+T_{c_{2}}\left(f_{2}^{\star}\right) .
$$

In this paper, the speeds $c_{1}, c_{2}$ are assumed to be fixed by prior anatomical and electrophysiological knowledge.

This source separation problem is a difficult ill-posed inverse problem, because the number of parameters (two space/time sources) to be estimated is vastly larger than the dimensionality of the observations. This difficulty is further increased by the presence of noise (biological and instrumental) and because the linear mappings $T_{c_{i}}$ might degrade some of the information present in the sources. This section thus defines constraints on the sources to ensure uniqueness of the decomposition (5) in the noiseless case $W=0$, and stability when noise is added to the measurements.

\subsection{Sources Localization Constraints}

Even in the noiseless case $W=0$, the separation (5) is not always unique. To recover uniqueness, we impose that each source $f_{i}$ is localized in space in a fixed domain $\Omega_{i}$ such that $\Omega_{1} \cap \Omega_{2}=\emptyset$. We thus defines the following set of admissible sources

$$
F_{i}=\left\{f \in \mathcal{C}^{2}(E): x \notin \Omega_{i} \Rightarrow f(t, x)=0\right\} .
$$

The constraint $f_{i} \in F_{i}$ makes sense from the observations of Per Roland et al. [5], that show that $f_{1}$ is localized near the cortical retinotopic impact center of the stimulus, whereas $T_{c_{2}}\left(f_{2}\right)$ originates from a surrounding cortical area 19 or 21. The following theorem, whose proof is given in [6] shows that the constraints $f_{i} \in F_{i}$ ensures uniqueness of the decomposition if $W=0$.

Theorem 3.1. If $c_{1}, c_{2} \in \mathbb{R}_{+}$and $f_{c_{i}}, f_{c_{i}^{\prime}} \in F_{i}$, then

$$
\begin{gathered}
T_{c_{1}}\left(f_{c_{1}}\right)+T_{c_{2}}\left(f_{c_{2}}\right)=T_{c_{1}}\left(f_{c_{1}^{\prime}}\right)+T_{c_{2}}\left(f_{c_{2}^{\prime}}\right) \\
\Longrightarrow f_{c_{1}}=f_{c_{2}}=f_{c_{1}^{\prime}}=f_{c_{2}^{\prime}}=0 .
\end{gathered}
$$

\subsection{Sources Smoothness Constraints}

To remove the noise while performing the separation, we assume that each source $f_{i}$ is smooth, and impose that the following Sobolev energy is small

$$
J\left(f_{i}\right)=\left\|\frac{\partial f_{i}}{\partial x}\right\|^{2}+\left\|\frac{\partial f_{i}}{\partial y}\right\|^{2}+\eta\left\|\frac{\partial f_{i}}{\partial t}\right\|^{2}
$$

The parameter $\eta$ defines the space/time scaling of the source, and is estimated by anatomical and functional knowledge. 


\section{TRAVELING COMPONENT ANALYSIS}

Constraints (6) and (7) are integrated in an optimization problem that allows us to recover the sources.

\subsection{Variational Formulation}

The VSDI signal is acquired on a small portion $U \subset \mathbb{R}^{2}$ of the visual cortex, that has an hexagonal shape of diagonal length $4.2 \mathrm{~mm}$, as shown on Figure 1. The propagating waves are however assumed to propagate on the whole plane $\mathbb{R}^{2}$. We thus impose the measurement model (4) only for points $x \in U$.

Using the orthogonal projector on a set $A \subset \mathbb{R}^{2}$

$$
P_{A}(f)(t, x)=\left\{\begin{array}{l}
f(t, x) \text { if }(t, x) \in \mathbb{R}^{+} \times A, \\
0 \text { otherwise, }
\end{array},\right.
$$

where $f \in \mathcal{C}^{2}(E)$. the separation (5) is obtained by integrating constraints (6) and (7) in a penalized least square

$$
\begin{aligned}
\min _{\tilde{f}_{1}, \tilde{f}_{2}} \| P_{U}\left(X-T_{c_{1}} P_{\Omega_{1}} \tilde{f}_{1}-\right. & \left.T_{c_{2}} P_{\Omega_{2}} \tilde{f}_{2}\right) \|^{2} \\
& +\lambda_{1} J\left(\tilde{f}_{1}\right)+\lambda_{2} J\left(\tilde{f}_{2}\right)
\end{aligned}
$$

The parameters $\lambda_{i}$ should be adapted carefully depending both on the noise level and on the expected smoothness of the sources.

Although the observations $X$ are available only inside $U$, the sources are retrieved on the whole domain. Reconstructing a signal $X^{\star}=T_{c_{1}} f_{1}^{\star}+T_{c_{2}} f_{2}^{\star}$ realizes both a denoising and an inpainting calculus, since one extrapolates the data outside $U$.

\subsection{Discrete Algorithm}

The application of the continuous method described in Section 2 to biological measurements requires the discretization of the model (analagous to that performed by the sensor matrix of the VSD camera). Both the observations $X$ and the sources $f_{i}$ are discretized on an uniform spatio-temporal grid of size $N_{t} \times N_{x}^{2}$. The propagation is computed using a discretized operator $T_{c}$, that is obtained by solving the PDE (3) with finite differences.

Solving (8) corresponds to the minimization of a quadratic functional over the discrete grid, which can be solved using a conjugate gradient descent. This process requires the computation of the adjoint operator $T_{c_{i}}^{\star}$ that satisfies $\left\langle T_{c_{i}}^{\star} u, v\right\rangle=$ $\left\langle u, T_{c_{i}} v\right\rangle$ for the discrete inner product on the computation grid. This requires the resolution of a wave equation that propagates backward in time.

\section{NUMERICAL RESULTS}

\subsection{Synthetic Data}

We evaluate numerically the efficiency of our traveling component analysis by applying it to synthetic data where the ground trust sources are known. This synthetic model aims at being close to the observation of Per Roland et al. [5] described in section 1.2.

In the following, $U$ is an hexagon of diagonal length 4.2. To study the influence of the size of the supports $\Omega_{i}$, we consider for $i=1,2$ a family of sources $f_{i}^{r}=P_{\Omega_{i}(r)} f_{i}$ defined over a domain $\Omega_{i}(r)$ of width $r \geqslant 0$, as shown the figure on the right

$$
\Omega_{i}(r)=\left\{x \in \mathbb{R}^{2}: \min _{y \in \Omega_{i}}\|x-y\| \leqslant r\right\}
$$

where $\Omega_{1}=\left\{x_{1}, x_{2}\right\} \subset \mathbb{R}^{2}$ and $\Omega_{2}=\left\{(x, y) \in \mathbb{R}^{2} \mid x^{2}+y^{2}=R^{2}\right\}$, where $R=8$.

The source $f_{1}$ is a sum of two gaussians of standard deviation 0.5 localized at the two points of $\Omega_{1}$. Source $f_{2}$ is a sum of gaussians of standard deviation 0.2 localized at all points

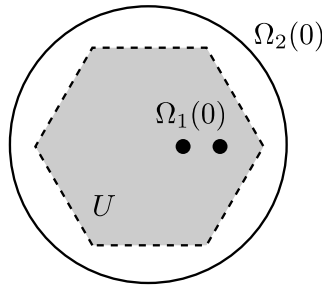
of the curve $\Omega_{2}$.

Moreover, $\left\|T_{c_{1}}\left(f_{1}\right)\right\|_{\infty}=1,\left\|T_{c_{2}}\left(f_{2}\right)\right\|_{\infty}=1 / 10, f_{1}$ and $f_{2}$ are of opposite signs. We consider a discrete domain of size $N_{t}=46, N_{x}=101$.

We fix the propagation speeds to $c_{1}=1.5$ and $c_{2}=$ $10 \times c_{1}$, and generate the observations $X=X_{r}$ according to the model (4) $X_{r}=T_{c_{1}}\left(f_{1}^{r}\right)+T_{c_{2}}\left(f_{2}^{r}\right)+W$ where $W$ is a gaussian white noise with variance $\sigma^{2}=10^{-3}$.

For each $r>0$, we apply the traveling component analysis algorithm described in Section 4.2 to recover sources $f_{i}^{r, \star}$ that solve (8), and compute the following recovery error

$$
\mathcal{E}(r)=\frac{\left\|f_{1}^{r}-f_{1}^{r, \star}\right\|}{\left\|f_{1}^{r}\right\|}+\frac{\left\|f_{2}^{r}-f_{2}^{r, \star}\right\|}{\left\|f_{2}^{r}\right\|}
$$

The parameters $\lambda_{1}$ and $\lambda_{2}$ are chosen in an oracle manner, to minimize the recovery error (10).

As the width $r$ increases, the recovery problem becomes increasingly ill-posed, so that the error $\mathcal{E}(r)$ increases. Ultimately for large enough $r$, the sets $\Omega_{i}(r)$ overlap and the uniqueness result of Theorem 3.1 does not holds anymore.

Figure 3 shows that even in this noisy setting, our algorithm performs well for a constraint domain of width smaller than $r=1$ and we obtain $\mathcal{E}(1)=0.3$.

\subsection{VSDI Data}

We apply our traveling wave separation algorithm on the VSDI data shown on figure 1, that were studied in [5]. We use the supports constraint $\Omega_{i}\left(r_{i}\right)$ defined in (9), and choose $r_{1}=1.5$ and $r_{2}=0.5$, that works well in the synthetic experiments shown in Figure 2 while being in agreement with physiological knowledges. Points $x_{1}$ and $x_{2}$ in subsection 5.1 correspond to the retinotopical centers. $U$ is an hexagon with a diagonal of length 4.2 and $R=8$. 

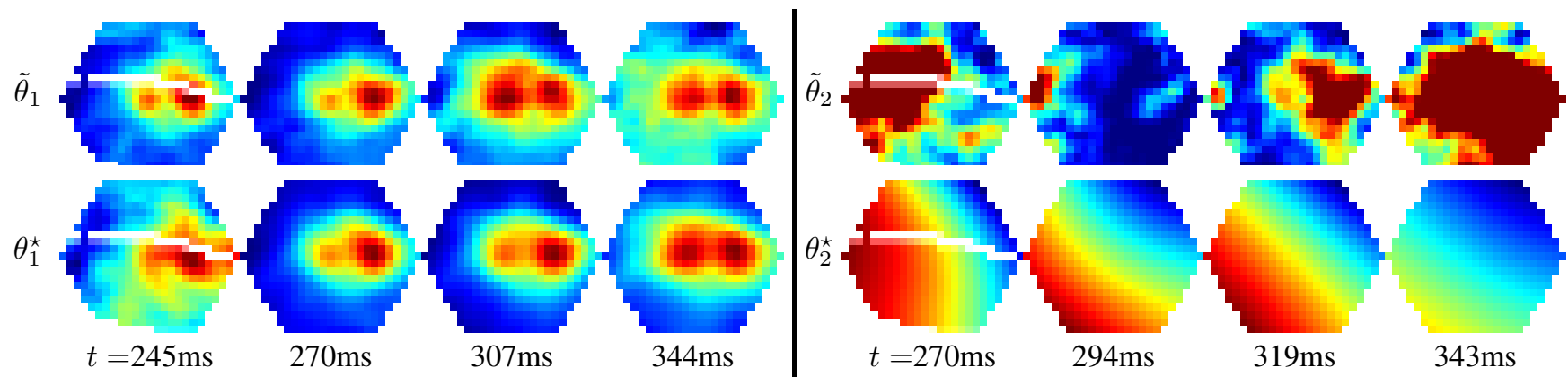

Fig. 2. Comparaison between the separation $\left(\tilde{\theta}_{1}, \tilde{\theta}_{2}\right)$ obtained with the method of [5] defined in (1) and $\left(\theta_{1}^{\star}, \theta_{2}^{\star}\right)$ obtained with our method. Left side. Top row: $\tilde{\theta}_{1}$. Bottom row: $\theta_{1}^{\star}$. Values range from blue $=-0.0039$ to red $=0.0155$. Right side. Top row: $\tilde{\theta}_{2}$. Bottom row: $\theta_{2}^{\star}$. Values range from blue $=-0.0024$ to red=0.0020).

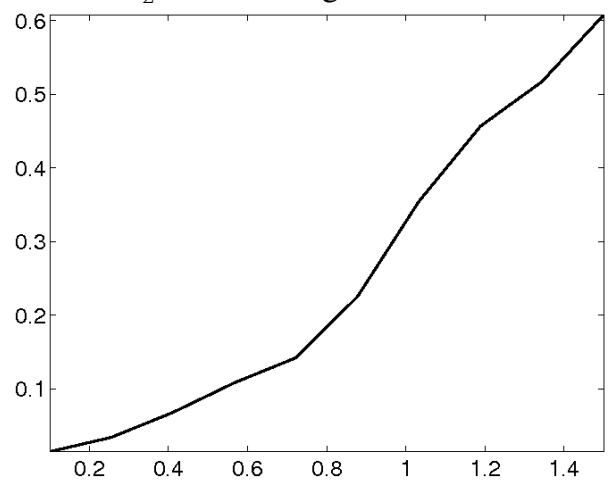

Fig. 3. Curve of recovery error $\mathcal{E}(r)$ as a function of $r$.

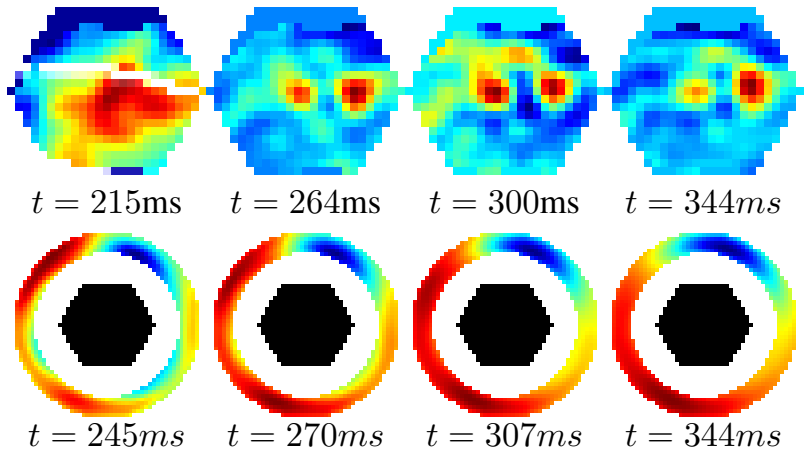

Fig. 4. Leftmost three images: source $f_{1}^{\star}(t, x)$ for several times $t$ (blue $=-1.3701$ to red $=2.2228$ ). Rightmost image: source $f_{2}^{\star}(t, x)$ for a single time $t$ the black hexagon shows the domain $U$ (blue $=-5.5823$ to red $=1.5545$ ).

The input data $X$ has been denoised prior to applying our method, by considering a signal averaged over 10 repeated trials. We impose large regularization parameters $\lambda_{1}$, $\lambda_{2}$ fixed such that $\left\|X-X^{\star}\right\| /\|X\| \approx 0.14$ to cope with imperfections in our model and ensure the recovery of smooth sources. Moreover, we fit $\lambda_{1}, \lambda_{2}$ to give a good visual result on both sources. Figure 1, bottom row, shows that the residual $X-X^{\star}$ has a small amplitude, which indicates that our model is able to efficiently represent the data with localized sources. Figure 2 compares the separation obtained by our approach and with the simpler thresholding (1). Our method confirms the existence of a feedback waves that propagates in the same direction as described in [5]. We achieve a full separation and retrieve smooth waves which is not possible with the thresholding (1). Figure 4 shows that our method estimates localized sources $\left(f_{1}^{\star}, f_{2}^{\star}\right)$ with a complicated temporal dynamic. We observed empiricaly a good separation for a ratio of $c_{1} / c_{2}=10$ which differs from the findings of [5].

\section{CONCLUSION/PERSPECTIVE}

This paper proposed a mathematical model of cortical traveling waves that integrates several plausible biological constraints. This allows to better represent the propagating components and to recover the sources of the activity. We applied our method on both synthetic data and real biological data. In future works, we would like to improve the separation process by finding optimal speeds that could be integrated into our variational optimization.

\section{REFERENCES}

[1] A. Grinvald and R. Hildesheim, "VSDI : A new era in functional imaging of cortical dynamics," Nature Reviews Neuroscience, vol. 5, no. 11, 2004.

[2] K. P. Hoffmann and J. Stone, "Conduction velocity of afferents to cat visual cortex: a correlation with cortical receptive field properties," Brain Res, vol. 32, no. 2, pp. 460-466, 1971.

[3] Y. Frégnac, P. Baudot, F. Chavane, J. Lorenceau, O. Marre, C. Monier, M. Pananceau P. Carelli, and G. Sadoc, Chapter 4, Multiscale Functional Imaging in V1 and Cortical Correlates of Apparent Motion, Springer-Verlag (US U. Ige and G. Masson), 2009.

[4] L.G. Nowak and J. Bullier, "The timing of information transfer in the visual system," Plenum Pub Corp, vol. 12, no. 11, pp. 205-241, 1997.

[5] P. E. Roland, A. Hanazawa, C. Undeman, D. Eriksson, T. Tompa, H. Nakamura, S. Valentiniene, and B. Ahmed, "Cortical feedback depolarization waves: A mechanism of top-down influence on early visual areas," PNAS, vol. 103, no. 33, pp. 12586-12591, 2006.

[6] N. Schmidt, "Separation of traveling waves in cortical networks using optical imaging," Master thesis, École Normale Supérieure de Cachan, 2009. 